\title{
Projet GAP \\ (Guneydogu Anadolu Projesi) Anatolie du sud-est (Turquie)
}

\author{
The GAP projet (Guneydogu Anadolu Projesi) \\ in south-east Anatoly (Turkey)
}

par M. Mesny

Ingénieur général du Génie rural, des Eaux et des Forêts

The GAP Project (Guneydogu Anadolu Projesi) in Turkey is the most important regional development project in the world. It concerns the catchments of Euphrate and Tigre rivers. The total cost of the infrastructures is estimated at U.S. $\$ 32$ billions, with 22 dams (the largest one is ATATURK dam $-48,7$ billions $m^{3}$ and $2400 \mathrm{MW}$ - in operation since 1993) and 19 hydropower stations. 1,78 million hectares will be irrigated.

With the GAP Project, Turkey will increase its power production by $35 \%$ and its irrigated area by more than $50 \%$. For Turkey this project is politically important, as the concerned area has a majority of Kurd population. But it is also a cause of tension in the Middle East, where water is a disputed resource, between Turkey and Syria and Irak.

\section{I — PRÉSENTATION GÉNÉRALE}

Le projet GAP concerne $75000 \mathrm{~km}^{2}$ et 6 millions d'habitants, soit $10 \%$ de la surface de la Turquie et $10 \%$ de sa population. La population y est en majorité d'origine kurde avec un taux de croissance démographique de $3,5 \%$, contre moins de $2 \%$ pour l'ensemble de la Turquie.

\begin{tabular}{|l|c|c|c|c|}
\cline { 2 - 5 } \multicolumn{1}{c|}{} & Euphrate & Tigre & $\begin{array}{c}\text { Total } \\
\text { projet } \\
\text { GAP }\end{array}$ & $\begin{array}{c}\text { Actuel } \\
\text { Turquie } \\
\text { (hors GAP) }\end{array}$ \\
\hline $\begin{array}{l}\text { Irrigation } \\
\left(10^{6} \text { ha) }\right.\end{array}$ & 1,15 & 0,63 & 1,78 & 3,3 \\
\hline $\begin{array}{l}\text { Production } \\
\text { hydro-électrique } \\
\left(\mathrm{GWh}^{1} \text { /an) }\right.\end{array}$ & 20000 & 7000 & 27000 & $\begin{array}{c}80000 \\
(1994)\end{array}$ \\
\hline
\end{tabular}

$1 \mathrm{GWh}=$ giga watt heure $=10^{9}$ watt heure $\mathrm{MW}=$ mega watt $=10^{6}$ watt
C'est actuellement le plus grand projet de développement régional en construction dans le monde. Son coût total, en ce qui concerne les infrastructures, est estimé à 32 milliards \$ dont 13 ont déjà été mobilisés et dépensés. Le rythme actuel de dépenses est de 600 à 700 millions $\$ /$ an, ce qui correspond à un achèvement des infrastructures dans une trentaine d'années, vers 2030.

Le projet GAP, c'est 22 barrages (14 dans le bassin de l'Euphrate et 8 dans le bassin du Tigre) et 19 centrales hydroélectriques (11 sur l'Euphrate et 8 sur le Tigre) correspondant à une puissance installée totale de $8500 \mathrm{MW}^{1}$ dont 4200 sont déjà en production. Il permettra l'irrigation de 1,78 million d'hectares.

\section{RESSOURCES EN EAU}

\subsection{Barrage ATATURK sur l'Euphrate}

Ce barrage en enrochements achevé en 1993 est actuellement le $6^{\text {eme }}$ plus grand barrage au monde pour le volume de la digue $\left(84,5\right.$ millions $\left.\mathrm{m}^{3}\right)$ : 
1. Vue d'ensemble du barrage d'Ataturk.

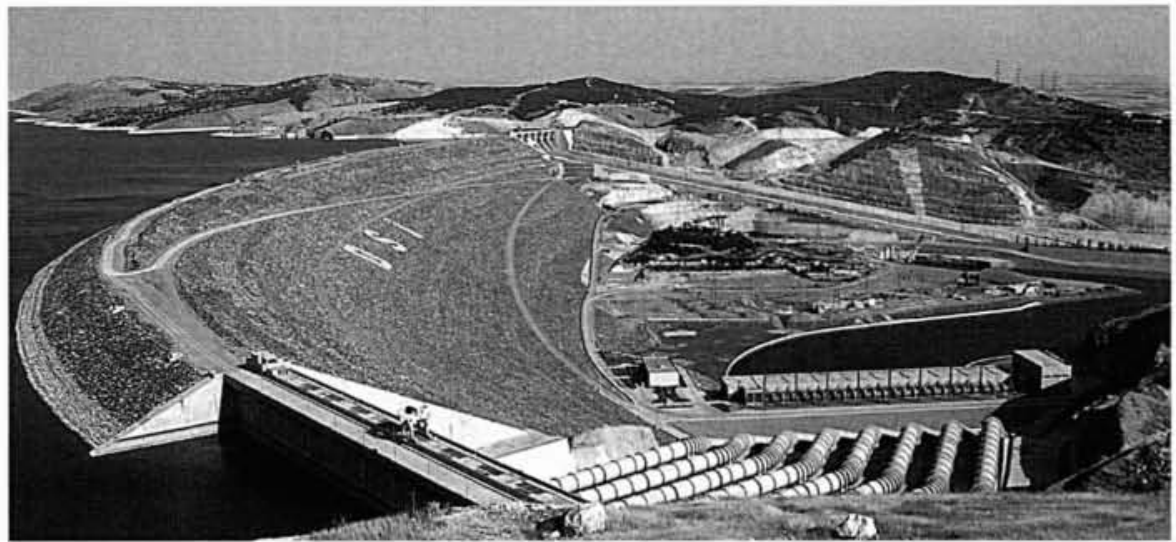

\begin{tabular}{|c|c|c|c|c|c|c|}
\hline Barrage & $\begin{array}{c}\text { Capacité } \\
10^{9} \mathrm{~m}^{3}\end{array}$ & Usage & $\begin{array}{c}\text { Puissance } \\
\text { installée MW }\end{array}$ & $\begin{array}{c}\text { Production } \\
\text { GWh/an }\end{array}$ & $\begin{array}{c}\text { Surface à } \\
\text { irriguer hectares }\end{array}$ & Commentaires \\
\hline KEBAN & & hydroélectricité & 1360 & 5300 & & terminé en 1974 \\
\hline KARAKAYA & 9,37 & hydroélectricité & 1800 & 7300 & & terminé en 1988 \\
\hline ATATURK & 48,7 & $\begin{array}{l}\text { hydroélectricité } \\
+ \text { irrigation }\end{array}$ & 2400 & 9000 & 882000 & terminé en 1993 \\
\hline BIRECIK $^{2}$ & & $\begin{array}{c}\text { hydroélectricité } \\
+ \text { irrigation }\end{array}$ & 670 & 2500 & 65000 & construction 1996-2001 \\
\hline KARKAMIS & & hydroélectricité & 180 & 650 & & $\begin{array}{c}\text { démarrage de la } \\
\text { construction en mai } 1996\end{array}$ \\
\hline
\end{tabular}

Tableau 1.- Principaux barrages sur l'Euphrate

- volume de la retenue (niveau normal) : 48,7 milliards $\mathrm{m}^{3}$;

- volume utile (entre le niveau normal et le niveau minimal de production hydro-électrique) : 18 milliards $\mathrm{m}^{3}$;

- usine hydroélectrique : $2400 \mathrm{MW}$ installés - production annuelle : $9000 \mathrm{GWh}$.

Une entreprise française SOLETANCHE est intervenue lors de la construction du barrage.

Le barrage ATATURK est l'élément principal d'une chaîne de barrages sur l'Euphrate, dont l'objectif est double : la production hydroélectrique de $20000 \mathrm{GWh}(6450 \mathrm{MW})$ et l'irrigation de 1,15 million d'hectares.

D'amont en aval, les principaux barrages sur l'Euphrate figurent sur le tableau 1.
La réserve utile de cette chaîne de barrages est de 42 milliards $\mathrm{m}^{3}$, alors que les apports moyens annuels (1937-1980) de l'Euphrate à la frontière syrienne s'élèvent à 30,8 milliards $\mathrm{m}^{3}$.

\subsection{Le Tigre}

3 barrages sont en construction (tableau 2).

5 autres barrages sont en projet (Silvan, Kayser, Garzan, Ilisu, Cizre).

Sur le Tigre, le projet du GAP prévoit :

- hydroélectricité : puissance installée : $2200 \mathrm{MW}$ production annuelle : $7000 \mathrm{GWh}$

- irrigation : 630000 hectares.

\begin{tabular}{|c|c|c|c|c|c|}
\hline Barrage & $\begin{array}{l}\text { Capacité } \\
10^{9} \mathrm{~m}^{3}\end{array}$ & Usage & $\begin{array}{c}\text { Puissance } \\
\text { à installer MW }\end{array}$ & $\begin{array}{c}\text { Production } \\
\text { prévue GWh/an }\end{array}$ & $\begin{array}{c}\text { Surface à } \\
\text { irriguer hectares }\end{array}$ \\
\hline KRALKIZI & 1,92 & $\begin{array}{c}\text { hydroélectricité } \\
\text { + irrigation }\end{array}$ & 94 & 145 & 126000 \\
\hline DICLE & & $\begin{array}{l}\text { hydroélectricité } \\
+ \text { irrigation }\end{array}$ & 110 & 300 & ) \\
\hline BATMAN & 1,175 & $\begin{array}{l}\text { hydroélectricité } \\
+ \text { irrigation }\end{array}$ & 198 & 480 & 38000 \\
\hline
\end{tabular}

Tableau 2. - Barrages sur le Tigre

\footnotetext{
${ }^{2}$ Coyne et Bellier et Alstom interviennent sur le barrage de Birecik qui fait l'objet d'un B.O.T. (Build Operate Transfer).
} 


\subsection{Eaux souterraines}

Le potentiel en eaux souterraines est important dans les plaines de Sanli Urfa, Mardin et Diyarbakir. Ces eaux souterraines sont utilisées pour l'alimentation en eau potable et pour de petits périmètres d'irrigation, l'irrigation commençant ainsi à être pratiquée sans attendre l'arrivée des infrastructures d'adduction du GAP.

\subsection{Problèmes internationaux}

\subsubsection{L'Euphrate}

\section{(i) Les besoins de la Turquie}

98\% du débit de l'Euphrate provient de la Turquie qui ne représente pourtant que $28 \%$ du bassin versant. Le débit moyen annuel (1937-1980) de l'Euphrate à la frontière syrienne est de $975 \mathrm{~m}^{3} / \mathrm{s}\left(30,8\right.$ milliards $\left.\mathrm{m}^{3}\right)$. Avant les aménagements, le débit naturel variait de $120 \mathrm{~m}^{3} / \mathrm{s}$ à $6000 \mathrm{~m}^{3} / \mathrm{s}$ suivant la saison. La capacité totale utile des barrages turcs implantés sur l'Euphrate étant supérieure de $36 \%$ aux apports moyens annuels (voir ci-dessus $\$ 2.1$ ), on peut considérer que l'Euphrate est maintenant régularisé de façon interannuelle à environ $1000 \mathrm{~m}^{3} / \mathrm{s}$. Dans le cadre d'un accord temporaire avec la Syrie en juillet 1987, la Turquie s'est engagée à laisser passer au moins $500 \mathrm{~m}^{3} / \mathrm{s}$ à la frontière syrienne, soit la moitié du débit régularisé.

Les 1,15 million d'hectares dont l'irrigation est prévue en Turquie à partir de l'Euphrate demanderont un prélèvement de l'ordre de 11 milliards $\mathrm{m}^{3} / \mathrm{an}$, ou $350 \mathrm{~m}^{3} / \mathrm{s}$, qui correspond à $35 \%$ des apports de l'Euphrate mais reste inférieur au débit maximal régularisé de $500 \mathrm{~m}^{3} / \mathrm{s}$ que s'est attribué la Turquie.

\section{(ii) Les besoins de la Syrie}

La Syrie dispose de 3 barrages sur l'Euphrate :

- Tabqa 14,2 milliards $\mathrm{m}^{3}$,
- Al-Baqth barrage de régulation,
- Tishreen 1,9 milliard $\mathrm{m}^{3}$.

Conformément à l'accord de juillet 1987, la Syrie doit recevoir de la Turquie un débit régularisé d'au moins $500 \mathrm{~m}^{3} / \mathrm{s}$ ou 15,7 milliards $\mathrm{m}^{3}$. Un accord signé en 1990 entre la Syrie et l'Irak attribue à la Syrie 6,6 milliards $\mathrm{m}^{3}$ $(42 \%)$, laissant à l'Irak 9,1 milliards $\mathrm{m}^{3}(58 \%), 640000$ ha devaient être irrigués en Syrie à partir du barrage de Tabqa. mais seulement 240000 sont effectivement irrigués à cause de problèmes de salinisation; les projets syriens prévoient l'utilisation de 8,3 milliards $\mathrm{m}^{3}$ de l'Euphrate, ce qui est supérieur aux disponibilités résultant des accords précédents.

\subsubsection{Le Tigre (apports turcs)}

La Turquie ne contribue au débit du Tigre qu’à hauteur de $40 \%$, le reste provenant de ses affluents irakiens. Le débit moyen annuel (1946-1985) à la frontière irako-turque est de $530 \mathrm{~m}^{3} / \mathrm{s}$, soit 16,8 milliards $\mathrm{m}^{3} / \mathrm{an}$. L'irrigation de 630000 ha en Turquie à partir du Tigre correspondra à un prélèvement de 6 milliards $\mathrm{m}^{3} / \mathrm{an}$, laissant disponible pour I'Irak environ 10 milliards $\mathrm{m}^{3} / \mathrm{an}$ ou $300 \mathrm{~m}^{3} / \mathrm{s}$.

\subsubsection{L'Irak}

L'Irak est traversé par les cours inférieurs de l'Euphrate. venant de la Turquie et de la Syrie, et du Tigre venant de la Turquie ; ces deux fleuves se jettent dans le Golfe persique après s'être rejoints dans le Shatt al-Arab.

\section{(i) Les apports de l'Euphrate}

Nous avons vu plus haut que, compte tenu des $500 \mathrm{~m}^{3} / \mathrm{s}$ que la Turquie doit laisser passer à la frontière syro-turque et de

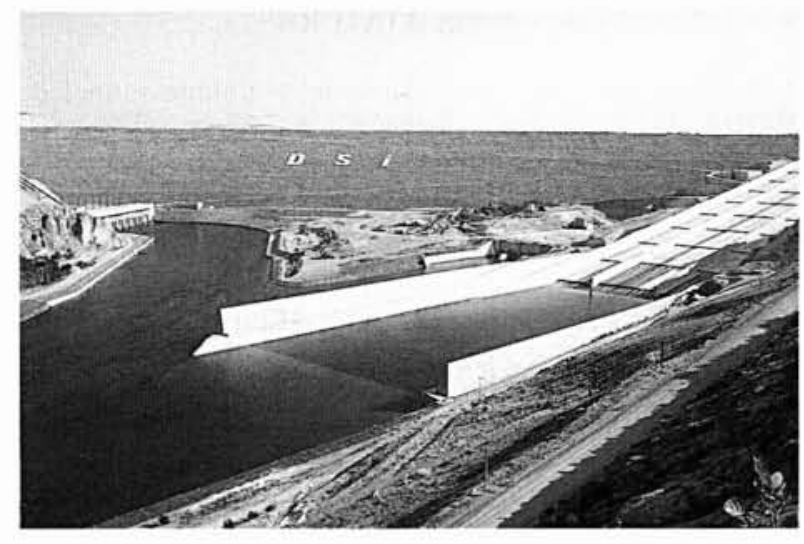

\section{Barrage d'Ataturk (photo Michel Mesny).}

l'accord de 1990 entre l'Irak et la Syrie, il restera disponible pour l'Irak 9 milliards $\mathrm{m}^{3}$ sur l'Euphrate en supposant un rejet nul dans le Shatt al-Arab. Mais cela ne permettra d'irriguer au mieux que la moitié des terres déjà irriguées en 1990, quand l'Irak recevait la quasi-totalité des apports naturels de l'Euphrate, soit 30 milliards $\mathrm{m}^{3}$. L'Irak a d'ailleurs réalisé des aménagements importants sur l'Euphrate, en particulier les barrages de Haditah, Ramadi et Hindiyah.

\section{(ii) Les apports du Tigre}

Compte tenu des prélèvements du GAP, nous avons vu qu'il resterait disponible à la frontière irakienne 10 milliards $\mathrm{m}^{3}$, auxquels il y a lieu d'ajouter les 27 milliards $\mathrm{m}^{3}$ d'apports des affluents en Irak, soit un total de 37 milliards $\mathrm{m}^{3}$. L'Irak a construit un grand barrage sur le Tigre à $80 \mathrm{~km}$ en amont de Mossoul: le Saddam dam, d'une capacité de 11 milliards $\mathrm{m}^{3}$, mais ce barrage est fondé sur du gypse et sa «mort est annoncée» sauf travaux importants d'étanchéité (de l'ordre de 500 millions F), puisqu'il perd depuis sa mise en eau en 1986 jusqu'à 80 t de fondation par jour (le gypse se dissout dans l'eau).

\section{(iii) Les besoins globaux}

Compte tenu du GAP, les apports totaux en Irak s'élèveraient ainsi à 9 milliards $\mathrm{m}^{3}$ pour l'Euphrate (accord de 1990 entre la Syrie et l'Irak) et 37 milliards $\mathrm{m}^{3}$ pour le Tigre, soit un total de 46 milliards $\mathrm{m}^{3}$, alors que la consommation de l'Irak en 1984 s'élevait déjà à 43 milliards $\mathrm{m}^{3}$ pour l'irrigation et 1,3 milliard $\mathrm{m}^{3}$ pour l'alimentation en eau potable, soit un total de 44,3 milliards $\mathrm{m}^{3}$, ce qui ne laisse à l'Irak guère de possibilité d'augmenter sa surface irriguée.

Cette situation sera encore aggravée par la dégradation prévisible de la qualité de l'eau des cours inférieurs de l'Euphrate et du Tigre (salinité, chlorures, engrais, pesticides). De plus le Shatt al-Arab ne sera quasiment plus alimenté, ce qui entraînera l'assèchement des marais du Sud.

\subsubsection{Conséquences pour le GAP}

En l'absence d'accords définitifs entre la Turquie et la Syrie et l'Irak sur le partage des eaux de l'Euphrate et du Tigre, les financiers internationaux, en particulier la Banque mondiale, se sont retirés du projet GAP, dont le financement doit ainsi être assuré sur fonds turcs, ce qui risque de ralentir beaucoup la réalisation du projet.

\section{III — LES PROJETS D'IRRIGATION}

Sur les 1780000 hectares à irriguer, 1150000 doivent l'être à partir de l'Euphrate et 630000 à partir du Tigre. 


\subsection{A partir du barrage ATATURK}

1) Le barrage ATATURK alimente le double tunnel de SANLI URFA $\left(26 \mathrm{~km}\right.$ - diamètre $\left.2 \times 7,62 \mathrm{~m}-328 \mathrm{~m}^{3} / \mathrm{s}\right)$ qui doit permettre l'irrigation de la plaine de URFA-HARRAN et des régions de MARDIN et CEYLANPINAR, soit 476000 ha :

\begin{tabular}{|l|c|c|}
\hline Périmètre d'irrigation & Surface & Etat d'avancement \\
\hline URFA-HARRAN & 141000 ha & équipement terminé \\
$\begin{array}{l}\text { MARDIN et } \\
\text { CEYLANPINAR } \\
\text { périmètres gravitaires } \\
\text { périmètres } \\
\text { avec pompage }\end{array}$ & 185000 ha & en construction \\
\hline \multicolumn{1}{|c|}{ Total } & $\mathbf{4 7 6 0 0 0 ~ h a ~ h a ~}$ & en projet \\
\hline
\end{tabular}

2) D'autres périmètres seront alimentés directement à partir du réservoir ATATURK par pompage pour être irrigués par aspersion :

\begin{tabular}{|c|c|c|}
\hline Périmètre d'irrigation & Surface & Etat d'avancement \\
\hline SIVEREK - HILVAN & 160000 ha & en projet \\
BOZOVA & 70000 ha & en projet \\
SURUC & 128000 ha & en projet \\
$\begin{array}{c}\text { ADIYAMAN - } \\
\text { KAHTA (en partie) }\end{array}$ & 30000 ha & en projet \\
\hline Total & $\mathbf{3 8 8 0 0 0}$ ha & \\
\hline
\end{tabular}

3) Le périmètre d'irrigation de YAYLAK de 18000 ha sera alimenté par un tunnel en construction à partir du réservoir ATATURK.

4) La surface totale qui sera irriguée à partir du barrage ATATURK est ainsi de :

- A partir des tunnels de SANLI URFA 476000 ha

- Par pompage à partir du réservoir

ATATURK

388000 ha

- Yaylak 18000 ha

- 3.2 Autres grands projets d'irrigation dans le bassin de l'Euphrate

\begin{tabular}{|c|c|c|}
\hline Périmètre d'irrigation & Surface & Ressource en eau \\
\hline $\begin{array}{l}\text { ADIYAMAN - KAHTA } \\
\text { (pour la partie non alimentée à } \\
\text { partir du barrage ATATURK) }\end{array}$ & 48000 ha & $\begin{array}{l}\text { Barrage de Gamgazi en construction. } \\
\text { Barrages de Gömikan, Koçali, Sirimtas, Fatopasa, } \\
\text { Büyükçay et Kahta en projet. }\end{array}$ \\
\hline ADIYAMAN - GOKSU & 70000 ha & $\begin{array}{l}\text { projet de barrage de Cataltepe } \\
\text { (qui assurera aussi l'alimentation en eau de la ville } \\
\text { de Gaziantep). }\end{array}$ \\
\hline GAZIANTEP & $\begin{array}{c}90000 \text { ha } \\
\text { (dont } 50000 \text { ha par aspersion) }\end{array}$ & $\begin{array}{l}\text { Barrage de Hancagiz et son réseau d'irrigation de } \\
7000 \text { ha en opération. } \\
\text { Barrages de Birecik et de Kayacik } \\
\text { en construction } \\
\text { Barrage de Kemli en projet. }\end{array}$ \\
\hline Total & 208000 ha & \\
\hline
\end{tabular}

- 3.3 Périmètres d'irrigation privés dans le bassin de l'Euphrate

60000 hectares, dont la plus grande partie est déjà irriguée à partir des eaux souterraines.
3.4 Surface totale des projets d'irrigation dans le bassin de l'Euphrate

- A partir du barrage ATATURK

- Autres grands projets

- Périmètres privés

Total bassin de l'Euphrate dont 590000 ha en aspersion.
882000 ha 208000 ha 60000 ha

1150000 ha

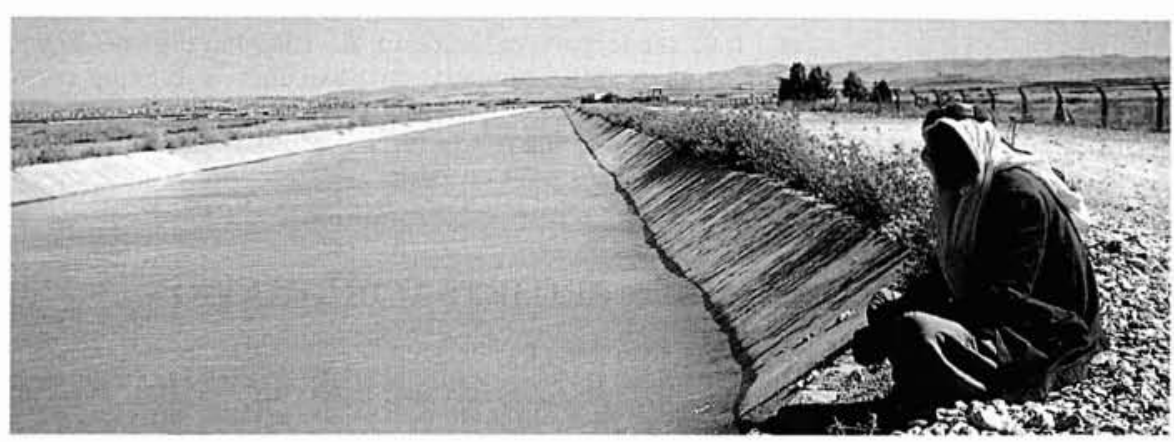

3. Canal d'irrigation. 
- 3.5 Bassin du Tigre

\begin{tabular}{|c|c|c|}
\hline Périmètre d'irrigation & Surface & Ressource en eau \\
\hline KRALKIZI & $\begin{array}{c}126000 \text { ha } \\
\text { (dont } 70000 \text { ha par aspersion) }\end{array}$ & $\begin{array}{l}\text { Barrages de Kralkizi et de Dicle } \\
\text { en construction. }\end{array}$ \\
\hline BATMAN & 38000 ha & Barrage de Batman en construction. \\
\hline BATMAN-SILVAN & $\begin{array}{l}255000 \text { ha } \\
\text { (dont } 60000 \text { ha par aspersion) }\end{array}$ & Barrages de Silvan et de Kayser en projet. \\
\hline GARZAN & 60000 ha & Barrage de Garzan en projet. \\
\hline ILISU & 4000 ha & Barrage hydroélectrique de Ilisu en projet. \\
\hline CIZRE & 121000 ha & Barrage de Cizre en projet. \\
\hline Périmètres privés & $\begin{array}{l}26000 \text { ha } \\
\text { effectivement irrigués }\end{array}$ & Eaux souterraines. \\
\hline Total bassin du Tigre & $\begin{array}{c}\mathbf{6 3 0} \mathbf{0 0 0} \text { ha } \\
\text { (dont } 130000 \text { ha en aspersion) }\end{array}$ & \\
\hline
\end{tabular}

\subsection{Taux de réalisation et mise en valeur}

Pour l'ensemble du projet GAP (Euphrate + Tigre) la situation en 1997 est la suivante :

\begin{tabular}{|l|c|c|c|c|}
\cline { 2 - 5 } \multicolumn{1}{c|}{} & $\begin{array}{c}\text { En } \\
\text { projet }\end{array}$ & $\begin{array}{c}\text { Construction } \\
\text { en cours }\end{array}$ & $\begin{array}{c}\text { En } \\
\text { opération }\end{array}$ & Total \\
\hline $\begin{array}{l}\text { Hydroélectricité } \\
\text { en MW }\end{array}$ & $\begin{array}{c}2000 \\
(27 \%)\end{array}$ & $\begin{array}{c}1300 \\
(17 \%)\end{array}$ & $\begin{array}{c}4200 \\
(56 \%)\end{array}$ & $\begin{array}{c}7500 \\
(100 \%)\end{array}$ \\
\hline Irrigation en ha & $\begin{array}{c}1363000 \\
(76 \%)\end{array}$ & $\begin{array}{c}284000 \\
(16 \%)\end{array}$ & $\begin{array}{c}133000 \\
(8 \%)\end{array}$ & $\begin{array}{c}1780000 \\
(100 \%)\end{array}$ \\
\hline
\end{tabular}

La surface effectivement irriguée en 1997 est détaillée ciaprès :

\section{- URFA - HARRAN \\ - GAZIANTEP \\ - Périmètres privés (Euphrate) \\ - Périmètres privés (Tigre)}

\section{Total}

L'irrigation est pratiquée par des agriculteurs, soit individuels, soit regroupés en Associations ou Unions d'irrigants. Il existe une vingtaine de ces Unions. La principale culture irriguée est le coton.

La mise en valeur agricole est lente, puisque si l'on retire les irrigations privés, qui sont en général antérieures au projet du GAP et utilisent les eaux souterraines, on obtient à peine 60000 ha irrigués, soit $3,4 \%$ du projet.

A Sanli Urfa, un Institut de recherche, créé en 1976, dispose d'une station de recherche de 48 hectares, sur laquelle sont conduits des essais d'irrigation pour la recherche appliquée et la vulgarisation auprès des futurs irrigants.

\section{L'ORGANISATION DU PROJET GAP}

\subsection{Administration du GAP}

C'est une superstructure interministérielle et interprovinciale de mission. Elle est coiffée par un Conseil supérieur, présidé par le Premier ministre et comportant le ministre chargé du GAP, le ministre du Plan et le ministre des Travaux publics. Un Conseil de coordination associe les représentants locaux. Cette Administration du GAP, elle-même, comprend 200 salariés (120 à ANKARA et 80 à SANLI URFA), dont 30 diplômés de l'enseignement supérieur parmi lesquels 12 agronomes.

\section{- 4.2 La Direction générale des Travaux hydrauliques (DSI - « General Directorate of State Hydraulic Works »)}

La DSI est le maître d'ouvrage des infrastructures hydrauliques du GAP. D'une façon générale, elle est responsable pour tout aménagement supérieur à $500 \mathrm{l} / \mathrm{s}$.

\section{- 4.3 Les Services publics ruraux (GDRS - « General Directorate of Rural Services »).}

La GDRS dépend du Premier ministre et en est fière. Elle est chargée des infrastructures rurales et des aménagements à la ferme (barrages de moins de $15 \mathrm{~m}$ de haut, ouvrages de moins de $500 \mathrm{l} / \mathrm{s}$, voirie communale, équipement à la parcelle, nivellement, drainage). C'est de la GDRS que dépend l'Institut de Recherche de Sanli Urfa.

\subsection{Le ministère de l'Agriculture}

La Direction régionale de l'Agriculture est chargée de la vulgarisation et possède aussi un Institut de recherche à Akcakale. 


\section{- 4.5 Les insuffisances de cette organisation}

Sur le plan hydraulique, les ouvrages sont bien conçus et bien exécutés et le rythme d'équipement est soutenu. La DSI est un excellent bétonneur, comme le montre le taux de réalisation des barrages et de l'équipement hydroélectrique (cf. §3.6).

Par contre la mise en valeur agricole par l'irrigation est lente (cf. § 3.6). Les principales raisons en sont :

- La priorité au stade des aménagements a été donnée à l'hydraulique en tenant peu compte des contraintes du foncier et de l'agronomie.

- La situation foncière est peu propice à la mise en valeur, avec $8 \%$ des agriculteurs possédant $50 \%$ des terres.

- L'absence de nivellement des parcelles rend l'irrigation de surface difficile à pratiquer, avec des risques d'érosion et d'engorgement des sols.

- La GDRS et la Direction régionale de l'Agriculture sont des administrations beaucoup moins dynamiques et avec beaucoup moins de moyens que la DSI et elles ne se coordonnent pas.
Pour réduire ce décalage entre les rythmes d'équipement et de mise en valeur les agriculteurs sont incités à constituer des associations/unions d'irrigants, qui devraient se voir transférer les charges financières du fonctionnement et de l'entretien des ouvrages d'irrigation.

\section{V —CONCLUSION}

Le projet GAP correspond à un aménagement hydraulique gigantesque qui assure déjà la maîtrise des écoulements de l'Euphrate et bientôt de ceux du Tigre. Le GAP exploite déjà $4200 \mathrm{MW}$.

A terme, il permettra à la Turquie d'augmenter de $35 \%$ sa production d'électricité et de plus de $50 \%$ sa surface irriguée. C'est un projet politiquement important pour la Turquie, car il concerne une zone à majorité kurde. Mais ce projet est aussi une source importante de tension au MoyenOrient, où l'eau est une ressource disputée, entre la Turquie et la Syrie et l'Irak.

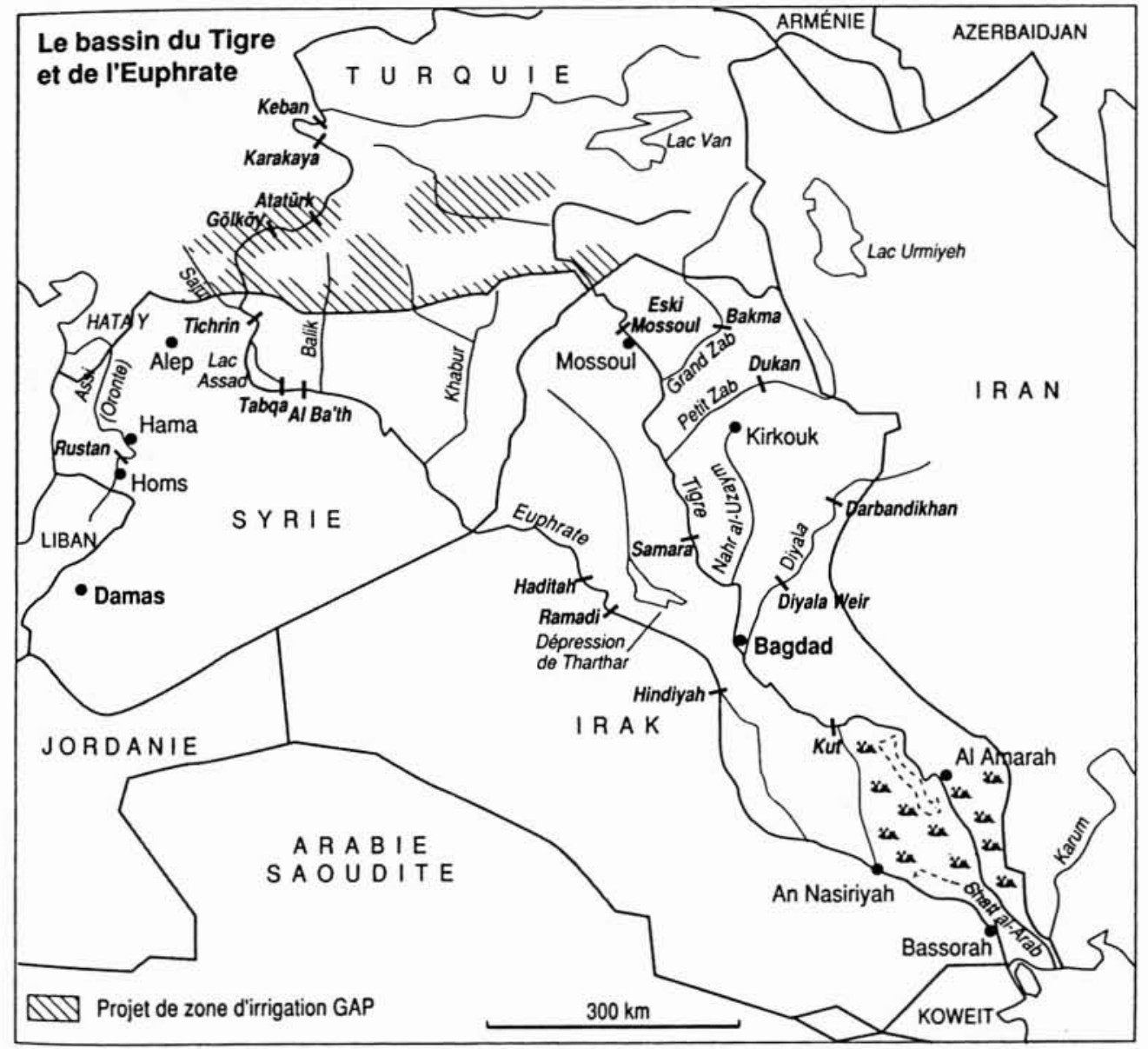

